\title{
A survey into COVID-19 Induced Pneumonia Detection and Feasibility of using UWB Medical Imaging
}

\author{
Nowshin Alam and Md. Abdur Rahman
}

\begin{abstract}
This paper presents a survey into the currently thriving research on using machine learning for COVID-19 induced pneumonia detection through the use of radiographic scans, presents a brief review of the methodologies and assesses the classification results, and finally presents an alternative in the form of ultrawideband (UWB) imaging. Few works on UWB imaging is investigated and used as a source of inspiration for developing an UWB imaging system for detection of accumulation of fluid in lungs. The goal is to extract information about dielectric property variation from backscattered UWB signals to detect pneumonia caused by COVID-19. An edge fed Vivaldi antenna along with a multilayer planar model for lung is simulated in CST microwave studio and subjected to UWB excitation. The backscattered signals in the form of S-parameters are analyzed with various Delay-and-Sum (DAS) algorithms and images are constructed for lung tissues of different permittivity and conductivity, where higher values are supported to allude to the infected lungs.
\end{abstract}

Index Terms - UWB imaging, UWB antenna, ultra wide band, microwave imaging, COVID-19 detection, Deep Learning.

\section{INTRODUCTION}

$\mathrm{C}$ ORONAVIRUS 2019 (COVID-19) pandemic is affecting countries all around the world as a leading cause for health complications and death. Originally referred to as Novel Coronavirus-Infected Pneumonia (NCIP), the COVID19 is highly infectious, and researchers are ardently working to develop more rapid, accurate and effective detection strategies to curb its spread. The COVID-19 testing methods currently available worldwide can be categorized into [1] [2]: a) molecular tests such as RT-PCR where respiratory tract swab samples undergo a relatively long and cumbersome process to transcribe RNA to DNA, and the presence of virus is detected in the amplified DNA using certain chemicals, b) antigen tests that detect certain proteins in fluid samples from nasal swabs, providing a faster result but more false negatives, c) antibody tests or serology tests that check for antibodies

Nowshin Alam is an Assistant Professor of the department of EEE, American International University-Bangladesh, 408/1, Kuratoli, Khilkhet, Dhaka 1229, Bangladesh. Email: nowshin.alam@aiub.edu.

Md. Abdur Rahman is a Professor of the department of EEE and the Associate Dean of Faculty of Engineering, American International University-Bangladesh, 408/1, Kuratoli, Khilkhet, Dhaka 1229, Bangladesh. Email: arahman@aiub.edu built up against COVID-19 in blood samples and thus cannot be useful for early detection of the disease. There have been studies into some more novel methods such as using breathalyzers [3] and sniff tests [4], but these methods need to be practically implemented in the field before their effectiveness can be judged. In addition to the diagnostic tests, an alternative approach employed by the doctors is radiographic examinations, such as chest X-Rays and CT scans from patients. The images are availed by the radiologists to search for visual indicators implying fluid accumulation and lung infection.

Due to the limitations in physical prototype development of new technologies during a time of worldwide lockdowns and social distancing measures, many researchers have focused their efforts on studying such medical scans and developing automated detection systems based on machine learning algorithms. Such methods are expected to aid in developing a triage method for patient risk management. Additionally, ultrawideband (UWB) imaging has drawn extensive attention researchers in the recent years due to its promise in highly accurate and precise localization as well as employing relatively smaller form factor antennas. It is one of the modalities of microwave imaging and an emerging short-range wireless technology characterized by brief pulses (below $2 \mathrm{~ns}$ ) and negligible power spread over a very large bandwidth (greater than $500 \mathrm{MHz}$ ) [5]. In the past, researchers have implemented microwave imaging as a possible low health risk method of medical imaging of human body through microwave tomography where the spatial distributions of dielectric properties closely computed by solving nonlinear inverse scattering problems [6]. UWB radar methods are computationally simpler and aim to only identify the presence and location of anomalies in the environment based on its distribution of dielectric properties. The former uses both the incident (transmitted) and scattered (received) fields, while the latter only uses reflected signals from the imaging object.

While microwave imaging has been used to detect a number of diseases in research literature, most of them are focused on the detection of solid objects such as tumors. The few instances of research focusing on detection of fluid accumulation in human organs have attempted to detect urine in bladder [7] or to identify pulmonary edema [8] or congestive heart failure using lamb lungs [9]. This paper continues the endeavor of our previous work [10] and focuses 
on studying the possibility of utilizing UWB imaging in order to detect and localize fluid accumulation in the lungs for COVID-19 induced pneumonia detection. Non-invasive identification of such lung anomaly would significantly aid in the discovery of complications in COVID-19 patient treatment as the lung air sacs of COVID patients get filled up by the fluid leaking from the blood vessels. The resultant inflammation may hinder the patients' oxygen absorption and thus cause difficulty in breathing. To avoid such cases from exacerbating into lung failure and even death in the worst-case scenario, any water accumulation in the lungs need to be identified as soon as possible. For this purpose, this paper integrates the recent literature regarding COVID-19 published since our previous review in Section II and revises the study of past microwave imaging applications to include UWB imaging system designs for additional kinds of medical uses in Section III. Based on the findings of section III and the methodology of UWB imaging methods explained in our previous work, Section IV presents our current system in development where an edge-fed Vivaldi antenna and a simulated human lung phantom have been designed in CST Microwave Studio and multiple imaging algorithms have been attempted for detecting the presence of water in the lung phantom. Section V concludes this work by identifying the limitations in the current prototype and suggesting future research directions.

\section{RESEARCH ON COVID-19 DETECTION}

The research community has experienced a recent thrust in investigations into COVID-19 detection using machine learning methods. By using artificial intelligence, automated methods attempt to deduce the presence of COVID-19 by identifying characteristic manifestations in the lung. Groundglass opacification (GGO) is one known feature of initial CT scans of covid patients, whereas consolidative opacities and crazy paving patterns can be found in more intermediate stages [11]. However, there is some difficulty in differentiating COVID-19 from other lung infections or viral pneumonia patients suffering from such diseases can sport similar patterns in CT scans. This is not a problem exclusive to CT scans but an issue that is prevalent in other detection methods based on imaging as well. Similar automated detection systems using X-ray images have also been proposed. There are certain benefits to using X-ray images to CT scans as they are more economical, easily accessible, and relatively less harmful for the body in terms of ionizing radiation. However, the early stage GCO manifestations viewable in $\mathrm{CT}$ may be missed by X-rays due to their lower resolution and overlapping of projections [1]. A flowchart of the general workflow of machine learning algorithms is presented in Fig. 1.

The classification results from recent literature that contributed to COVID-19 detection research are summarized

TABLE I

CLASSIFICATION RESULTS IN RECENT LITERATURE

\begin{tabular}{|c|c|c|c|c|c|c|c|}
\hline Source & Scans & Method & Data set (total) & $\begin{array}{l}\text { Training data } \\
\text { set (COVID) }\end{array}$ & $\begin{array}{l}\text { Test data set } \\
\text { (COVID) }\end{array}$ & $\begin{array}{c}\text { Internal } \\
\text { Validation } \\
\end{array}$ & External Validation \\
\hline $\mathrm{Xu}$ et al. [13] & CT & $\begin{array}{l}\text { ResNet }+ \\
\text { Location-attention }\end{array}$ & $\begin{array}{l}618 \text { images, } 219 \text { from } \\
110 \text { COVID patients }\end{array}$ & 189 & 30 & ---- & $\begin{array}{l}\text { SN: } 86.7 \%, \text { PPV: } \\
81.3 \%, \text { fl-score: } 83.9 \%\end{array}$ \\
\hline Song et al. [14] & CT & DRE-Net & $\begin{array}{l}1990 \text { images, } 777 \text { from } \\
88 \text { COVID patients }\end{array}$ & 466 & 233 & AUC 99\% & $\begin{array}{l}\text { AUC } 99 \%, \text { SN: } 93 \% \text {, } \\
\text { PPV: } 96 \%, \text { f1-score: } \\
94 \% \text {, A: } 94 \%\end{array}$ \\
\hline Wang et al. [21] & $\mathrm{CT}$ & $\begin{array}{l}\text { GoogleNet } \\
\text { Inception3 CNN }\end{array}$ & 1065 COVID-19 images & 320 & $\begin{array}{l}455 \text { (for } \\
\text { internal), } 290 \\
\text { (for external) }\end{array}$ & $\begin{array}{l}\text { A: } 89.5 \%, \text { SP: } \\
88 \%, \text { SN: } 87 \%\end{array}$ & $\begin{array}{l}\text { A: } 79.3 \%, \text { SP: } 83 \%, \text { SN: } \\
67 \% \text {, PPV: } 55 \% \text {, f1- } \\
\text { score: } 63 \%\end{array}$ \\
\hline $\begin{array}{l}\text { Zhang et al. } \\
\text { [18] }\end{array}$ & X-ray & CAAD & $\begin{array}{l}43370 \text { (X-VIRAL), } 213+ \\
509 \text { images of which } 106 \\
+493 \text { from COVID } \\
\text { patients (X-COVID + } \\
\text { Open-COVID) } \\
\end{array}$ & $\begin{array}{l}\text { No COVID- } \\
19 \text { data used } \\
\text { for training }\end{array}$ & ---- & ---- & $\begin{array}{l}\text { A: } 78.57 \%, \mathrm{SN}: \\
77.13 \%, \mathrm{SP}: 78.97 \% \text {, } \\
\text { AUC: } 84.43 \%\end{array}$ \\
\hline Wang et al. [16] & X-ray & COVID-Net & $\begin{array}{l}13975 \text { from } 13870 \\
\text { patients, } 358 \text { from } 266 \\
\text { COVID patients }\end{array}$ & 258 & 100 & ---- & $\begin{array}{l}\text { A: } 93.3 \%, \mathrm{SN}: 91 . \% \text {, } \\
\text { PPV: } 98.9 \%\end{array}$ \\
\hline Li et al. [19] & X-ray & COVID-GATNet & $\begin{array}{l}10192 \text { normal, } 7399 \\
\text { pneumonia, } 399 \text { COXID- } \\
19 \text { positive }\end{array}$ & 319 & 80 & ---- & $\begin{array}{l}\text { A: } 94.3 \%, \text { PPV: } 98.9 \% \text {, } \\
\text { SN: } 91.9 \%\end{array}$ \\
\hline Tabik et al. [20] & X-ray & COVID-SDNet & $\begin{array}{l}426 \text { COVID positive, } 426 \\
\text { negative images }\end{array}$ & 682 & 170 & ---- & $\begin{array}{l}\text { Accuracy: } 97.72 \pm \\
0.95 \% \text { (severe), } 86.90 \% \\
\pm 3.20 \% \text { (moderate), } \\
61.80 \% \pm 5.49 \% \text { (mild) }\end{array}$ \\
\hline $\begin{array}{l}\text { Tuncer et al. } \\
\text { [22] }\end{array}$ & X-ray & Cubic SVM & $\begin{array}{l}150 \text { normal and } \\
\text { pneumonia images, } 135 \\
\text { COVID }\end{array}$ & ---- & ---- & $\begin{array}{l}\text { A: } 97.01 \%, \mathrm{SN}: \\
97.09 \%, \mathrm{PPV}: \\
97.11 \%\end{array}$ & --- \\
\hline
\end{tabular}

A: Accuracy, SP: specificity, SN: sensitivity, AUC : Area Under Curve, Positive Predictive Value : PPV 
in Table I and the general summaries of the works described in the next subsection.

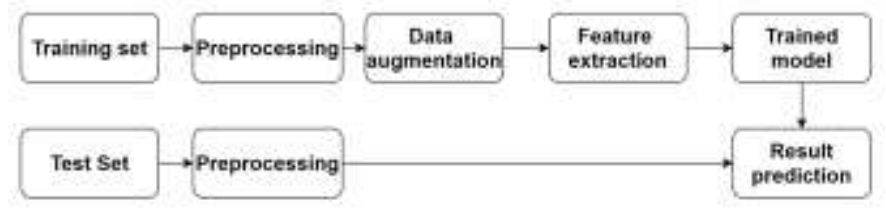

Fig. 1. General workflow of machine learning algorithms.

\section{A. Deep Learning Approach}

Li et al. [12] first proposed a 3D fully automated deep learning framework called COVNet that attempted to detect COVID-19 from chest CT scans collected from hospitals while trying to distinguish the results from Communityacquired pneumonia (CAP) and other afflictions such as lung nodules, chronic lung inflammation etc. The authors used a convolutional neural network (CNN) using RestNet50 as the backbone. They extracted lung regions as the Region of Interest (ROI) using UNet based segmentation and automatically generate localization maps using Gradientweighted Class Activation Mapping (Grad-CAM) method without manually annotating the data.

$\mathrm{Xu}$ et al. [13] designed an automated screening system for differentiating between COVID-19 and influenza-A viral pneumonia from a large dataset of CT scans collected from multiple hospitals. First a VNET20 based segmentation model was used to segment out infection regions, which were then operated on by a traditional ResNet-18 network structure alongside a modified model with location-attention mechanism. The overall result for a CT image was calculated using noisy-or Bayesian function.

Song et al. [14] introduced a fully automated deep learning lung CT diagnosis system called DeepPneumonia with automatic detection of main lesion features such as GGO. They developed Details Relation Extraction Neural Network (DRE-Net) based on pretrained ResNet50 for feature extraction, and utilized Feature Pyramid Network (FPN) and attention module to interpret network outputs. They set up a publicly available online server where CT images can be uploaded for online diagnosis. They also distinguished between COVID-19 related and bacterial pneumonia and detected the latter with an AUC of $99 \%$.

Hasan et al. [15] used CT images from free and open access datasets to develop a novel method for feature extraction where they used a handcrafted descriptor formed using QDeformed Entropy (QDE) in combination with deep learning features. A type of RNN called LSTM (Long Short-Term Memory) was used as the neural network classifier. They also discriminated between COVID-19 and pneumonia (not caused by coronavirus) with fairly high accuracy.

Wang, et al. [16] proposed an open-source CNN design called COVID-Net that can be publicly accessed in github. They also established an open access benchmark dataset containing X-ray images from 13,870 patients with the help of different public repositories. The architecture was pretrained on the ImageNet dataset and later a trained model was developed with the help of COVIDx dataset. It also employed the Adam optimizer and a learning policy of slowing down learning rate with learning stagnation. GSInquire was used as an explainability method to verify that COVID-Net made decisions based on actual features found in X-Ray images.

Turkoglu [17] used X-ray images from public datasets to build COVIDetectioNet, a COVID-19 diagnosis framework that commissioned a pretrained $\mathrm{CNN}$ based AlexNet architecture that was trained using transfer learning and utilized deep features from all layers including convolution layers. A relief feature selection algorithm allowed the reuse of information from pre-learned tasks and facilitated faster learning. The features were finally classified with the Support Vector Machine (SVM) classifier.

Zhang et al. [18] proposed the confidence-aware anomaly detection (CAAD) model that replaced the classifier by an anomaly detector by treating the non-COVID viral pneumonia cases as anomalies. The model comprises a shared feature extractor (EfficientNet pretrained on ImageNet), an anomaly detection module that assigned scores to X-ray images, and a confidence prediction module that reduced false negatives through re-assignment of the low confidence samples for further medical testing. The CAAD model was trained using on the in-house XVIRAL dataset. An interesting feature of this study was the detection of COVID-19 cases for the inhouse X-COVID and the public Open-COVID datasets despite the training dataset having no COVID-19 patient X-ray images. The model achieved an AUC of 83:61\% and 94.93\% respectively by recognizing the characteristic features COVID-19 as anomalies compared to the healthy or viral pneumonia samples.

The study by Li et al. [19] built a deep learning model named COVID-GATNet by combining the concepts of Dense Convolutional Network (DenseNet) and Graph Attention Network (GAT). The integrated collection of three public chest X-ray data sets containing COVID-19 positive images was expanded by scaling, rotation, brightness adjustment etc. DenseNet facilitated the reuse of features from previous layers and improve computational complexity. Multiple sets of independent attention mechanisms were also employed for better feature extraction.

Tabik et al. [20] developed a publicly accessible balanced database of COVID-19 images called COVIDGR-1.0 that comprised chest X-ray samples of four degrees of disease severity, ranging from mild symptoms to the most severe ones. The authors proposed a COVID Smart Data based Network (COVID-SDNet) methodology that uses a CNN based classifier based on Resnet-50 initialized with ImageNet weights and trained with a transfer learning method. Smart data was generated through preprocessing that combined noise elimination, segmentation-based cropping and data transformation. The scans were segmented with the U-Net segmentation model, both ReLU activation and SoftMax activation was utilized for the CNN and Stochastic Gradient Descent (SGD) was used as optimizer. One noteworthy contribution of their work was the establishment of a protocol on the selection and annotation method for the dataset images, 
created in collaboration with certified radiologists. The GradCAM method was used to justify the decisions made by the trained model, along with some visual explanation by means of a heat-map.

The study by Wang et al. [21] evaluated a deep learning algorithm using chest $\mathrm{CT}$ scans collected from hospitals and worked with radiologists to verify the accuracy of CT image feature extraction. After initial preprocessing involving Grayscale binarization, background area filling and color reversal, transfer learning was used with the help of modified inception model based on GoogleNet Inception3 CNN.

Tuncer et al. [22] presented a novel cognitive method for COVID-19 detection that did not require a number of parameters. Their approach employed a machine learning model called the exemplar model where chest X-ray scans from public datasets were put through fuzzy tree transformation, exemplar division and multi-kernel local binary pattern (MKLBP). Additionally, an iterative neighborhood component (INCA) was utilized for useful feature selection. The paper investigated 16 conventional classifiers, among which Cubic SVM achieved the highest $97.01 \%$ classification accuracy.

\section{B. Limitations}

While a significant number of COVID-19 detection methods involving $\mathrm{X}$-ray and computed tomography scans report high accuracy, certain roadblocks still exist in employing these approaches for the development of a clinical triage method. The most common concern is the possible health risks because of the exposure to ionizing radiation, which are significantly more severe in CT. A chest x-ray normally imposes approximately 0.01 rem ( 10 millirem) on the patient's body whereas the value $1 \mathrm{rem}(1,000 \mathrm{mrem})$ for a full-body CT scan [23]. New York state department [24] addresses the possible health risks due to prolonged or repeated radiation, which are especially significant for pregnant women and children. Medical screening equipment are becoming scarcer with the exponential rise in the number of cases across the world, and the availability of medical staff is declining as their heavy workload continues and many of them succumb to the disease themselves.

Additionally, the reported high accuracies and sensitivities are not always clinically significant as many datasets does not provide a complete spectrum of COVID-19 severity levels and predominantly focus on the most critical cases [20]. The machine learning methods based on datasets run the risk of failing to recognize low and moderate cases, which defeats the original purpose of early disease detection.

The stability of several models, in terms of standard deviation, was not calculated and the size of training and testing dataset was sometimes unclear or too small. Many datasets also lack collaboration between radiologists and AI experts and do not follow a consistent protocol in the annotation process of imaging scans [20]. Tabik et al. also attributed the high sensitivities in past works to the bias in the highly used COVID-19 Image Data Collection where the small number of COVID-19 positive cases (comprised mostly of late-stage patients) present contain heterogeneous data [20].

The emergence of newer mutated virus strains is also triggering a sizable amount of dataset shift, and the classification-based approaches may see a decline in performance as a result [18]. All of these factors together have so far prevented them from being considered as universally applicable long-term monitoring tool.

\section{RELEVANT RESEARCH ON MICROWAVE IMAGING}

UWB frequency spectrum ranges between $3.1-10.6 \mathrm{GHz}$ and can coexist with other wireless technologies with minimum interference. Its high resolution, moderately deep penetration in the human body, lack of unsafe ionizing radiation effects and antenna designs with comparatively small form factors have resulted in many researchers study the technology for medical imaging [25]. Our previous work [10] provided comparison among different UWB techniques for imaging lung for fluid accumulation purposes. To avoid repetition of information, this paper focuses on the information regarding implementation rather than the methodology of UWB imaging in the relevant papers.

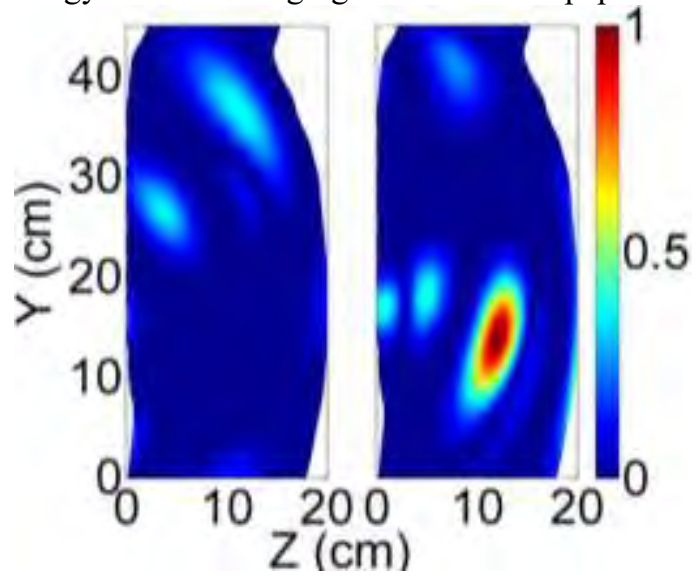

(a)

(b)

Fig. 2. Human torso phantom images containing (a) healthy lamb lungs and (b) lamb lungs that have been injected with 1 mililiter water. [26]

Rezaeieh et al.'s experimental work on pulmonary oedema detection through wideband antenna imaging can be considered the application of microwave imaging that contributed most significantly to detecting fluid accumulation in lungs and is particularly relevant to COVID-19 diagnosis. Through the imaging of a human torso phantom, they demonstrated the detection of water content as low as 1 milliliter. The proposed clinical system also performed admirably when conducted with healthy volunteers, although the result did not have significant clinical value due to the absence of lung ailments and the location of the heart.

The experimental setup included a portable Vector Network Analyzer (VNA) and an antenna array combined with a switching network. The antenna array was designed to operate in $0.7-1 \mathrm{GHz}$ band. S11 parameter of the optimum design managed to approach $-30 \mathrm{~dB}$ at resonant frequency $960 \mathrm{MHz}$. Data was collected from horizontally lying lung phantom or 
human volunteers in a multistatic data collection from the back of the torso.

This work suggested a novel frequency-domain imaging algorithm. First some preprocessing was performed to remove any reflections from skin due to the high permittivity of skin layer. For this purpose, the received electric field was calculated from the S-parameters measured by the VNA, following which the average value of these fields was calculated subtracted from each field. The fields were normalized and used to calculate the scattered electric field from each point $(\mathrm{x}, \mathrm{y})$ inside the imaged object.

$$
\begin{aligned}
& E_{s c c u r}^{n, m}(x, y)=E_{n o r m}^{n, m} \cdot J_{1}\left(k_{m} \rho\right) \cdot e^{h\left(k_{m i} \rho+\phi\right)} \\
& l(x, y)=\sum_{m}^{N_{f}} \sum_{n}^{N_{m}}\left|E_{s c m l}^{n, m}(x, y)\right|=\sum_{m}^{N_{f}} \sum_{n}^{N_{f}} E_{m, m, m}^{n, m}\left|J_{1}\left(k_{m} \rho\right)\right|
\end{aligned}
$$

Here $J_{l}()$ is Bessel function of the first kind of order zero, $k_{m}$ is the wavenumber of $m$-th frequency, $(\rho, \Phi)$ are the distance and the angle of each imaged point from the source, respectively. Despite the promising results, this work was not adopted for the method proposed in this paper as the experimental setup is difficult to replicate in the current situation of the world with COVID-19 pandemic related restrictions. Rezaeieh et al. [26] simulated the antenna in CST microwave studio, but the necessary data for imaging was collected experimentally without any simulation. Additionally, the artifact removal method is rather simple involving average subtraction and an average estimate of relative permittivity. No actual pulmonary edema patient was tested using the clinical system by the future works of their group, and the designed antenna did not qualify as an ultrawide antenna.

The next work that aided our design was Wang et al's [27] approach to breast cancer detection. While the end goal of their work was different, they designed a flexible UWB antenna array optimized for size and cost with a wearable design that may be applicable for lung imaging purposes as well. The antennas were first arranged in circular arrays, then assembled into 3 levels in groups of 4 to create a 3-D cylindrical array system. The antenna sports an omnidirectional radiation pattern with its $-10 \mathrm{~dB}$ bandwidth between 4.97 to $11.73 \mathrm{GHz}$ with two resonant frequencies at 5.7556 GHz (-25.386 dB) and 10.1 GHz (-29.085 dB). The maximum gain is found to fall between 2.5 to $4 \mathrm{~dB}$. The simulation was performed in CST using the time domain finite difference time (FDTD) solver, where an MRI-based breast model and a tumor of $5 \mathrm{~mm}$ diameter was placed in the software workspace. CST provided human tissue was used for the breast phantom layers and the "Biological Property Definition" macro was used to model the incidence dependent dielectric prosperities with the help of Cole-Cole expressions. Imaging results were generated by using the classic and robust Delay-and-Sum (DAS) algorithm.

Abdelhamid and Allam [28] implemented their proposed design for lung cancer detection practically and found satisfying degree of match between simulated and experimental characteristics of the antenna. They designed a cupcake UWB antenna using Rogers RO4350 (lossy) material as a substrate. The achieved frequency band lies within 2.9 $\mathrm{GHz}$ to $12 \mathrm{GHz}$. The authors used CST simulation for simulating comparing the S-parameters of the UWB antenna for simulated lung phantom models corresponding to different stages of lung cancer. No images for visualizing the cancerous regions were generated.

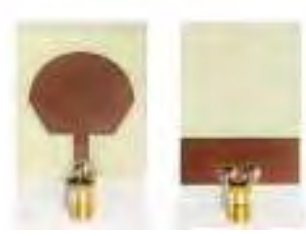

(a)

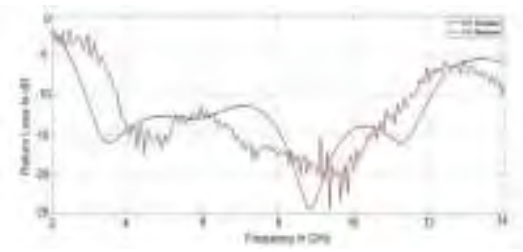

(b)
Fig. 3. (a) Implementation of cupcake antenna and (b) Simulated and measured S11 parameter by [28].

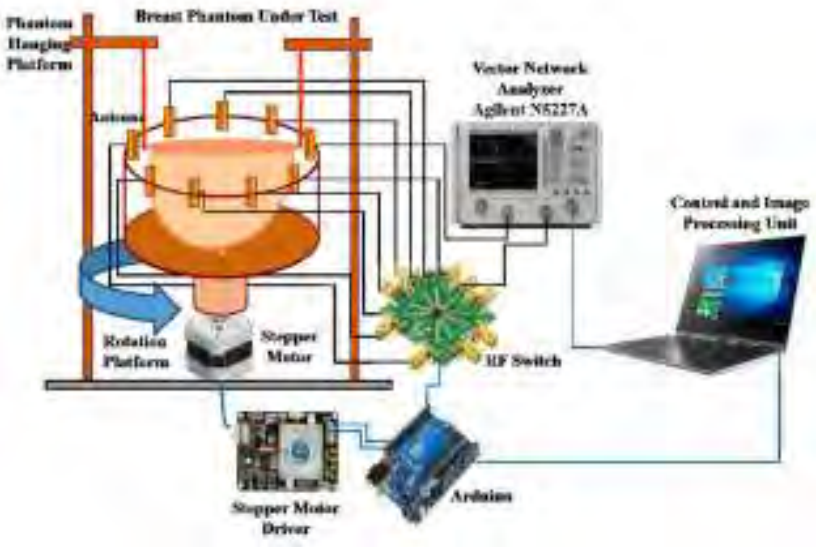

Fig. 4. Breast imaging system developed in [29].

Islam et al. [29] developed a computer controlled and microcontroller-based microwave imaging system where they employed a compact side slotted tapered slot antenna with radiating fins. They set up the prototype of a clinical system consisting of a mechanical rotating platform that houses a breast phantom subject to transmitted UWB signals from port 1 of an Agilent E8358A VNA. The receiving antennas arranged around the phantom are switched in order through the VNA and MATLAB program in PC and the backscattered data is captured by the VNA in the form of S-parameters. The designed antennas for this purpose were Vivaldi antennas with 2.80 to $7.00 \mathrm{GHz}$ bandwidth. After data collection, breast tumor detection was done through imaging with both DAS and a newly proposed Iteratively Corrected Delay and Sum (ICDAS) algorithm that demonstrated a slight increase in image clarity compared to DAS.

\section{Proposed Method}

The human tissues with differing relative permittivity reflect any UWB signal penetrating through the tissue layers, and the amount of attenuation that the UWB signal undergoes also varies across layers due to the uneven conductivity of the tissues. The information gained from studying the 
backscattered and the transmitted signals can thus be utilized to identify irregularities with different permittivity with respect to their neighboring tissues. This work attempts to build an imaging system that characterizes such dielectric property distribution by transmitting microwave pulse signals from a pair of antennas placed close (a few millimeters) to a human torso phantom, one in the front and one in the back.

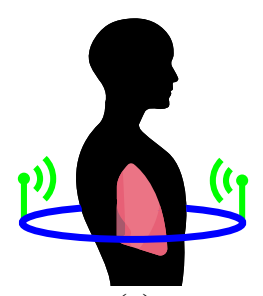

(a)

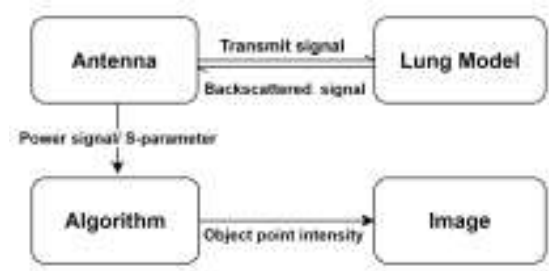

(b)
Fig. 5. (a) Antenna setup and (b) Block diagram for the proposed imaging system.

The antennas and the torso phantom were both simulated with the time domain solver using CST Studio Suite 2021 on a Windows 10 machine, using an AMD Ryzen $94900 \mathrm{H}$ processor with 16 GB RAM and NVIDIA GeForce RTX 2060 graphics card. The multilayer planar model [30] for the torso was derived from a simple human male model called "Duke" from an extension of the Virtual Human data set. A section of the Duke's anatomy passing through the heart has been utilized to build the model.

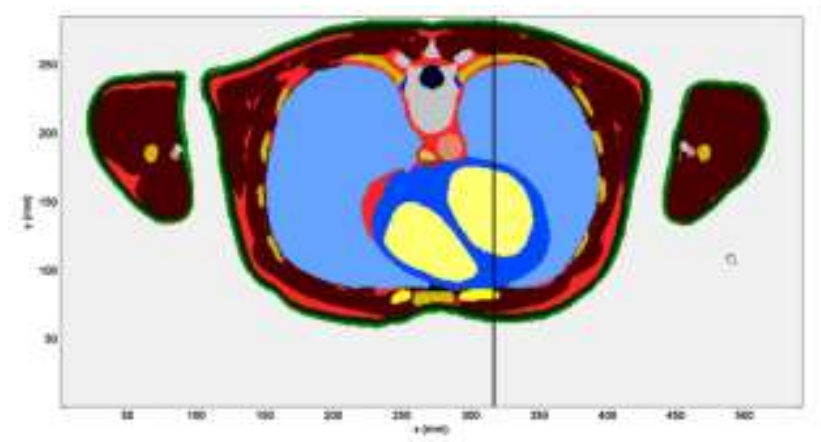

Fig. 6. Axial section of the Duke's anatomy for building the multilayer planar model [30].

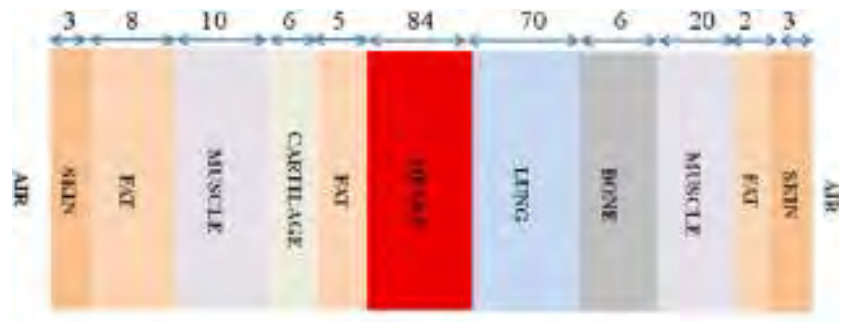

Fig. 7. Multilayer planar model developed from the Duke's anatomy [30].

The dielectric properties for the body tissues are taken from the human body voxel models in CST that sport frequency dependent permittivity and loss tangent characteristics. While modeling a lung that is affected by COVID-19 induced pneumonia, the lung layer in the model is modified to have higher conductivity and permittivity values compared to the case for normal lungs. An increase in conductivity and permittivity as well as a linear correlation between the mean of the maximum loss tangent and the lung water content is observed with increasing fluid content in rat lungs [31], so it is assumed that a similar pattern may be present in human lung.

The previous findings of Nopp et al. [32] also support this theory where they defined the ratio of the air volume and the volume of the condensed matters as filling factor $F$. The researchers found lower conductivity and permittivity in calf lung at higher values of $\mathrm{F}$ which correspond to higher air content (in other words, lower fluid content), and predicted an increase of conductivity and permittivity due to storage of liquid in alveoli during pneumonia. The experimental results approximated that at $100 \%$ air content the lung permittivity and conductivity was 43 and 0.05 , and decreased inversely proportional to $\sqrt{F}$ at other $\mathrm{F}$ values. We considered a lung $50 \%$ full of air and $50 \%$ full of fluid to have a permittivity and conductivity of 63.64 and instead of the 49.38 default value in CST, and conductivity of 0.5445 instead of the default 0.434 .

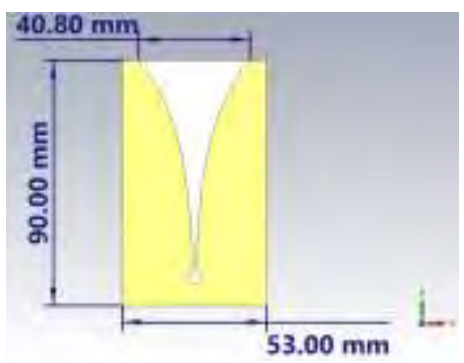

(a)

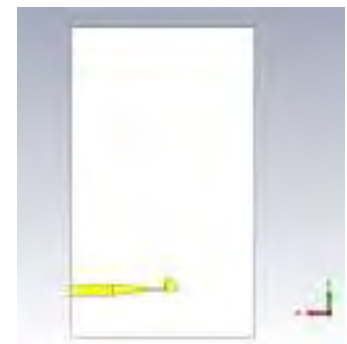

(b)
Fig. 8. (a) Front view and (b) Back view of Vivaldi antenna.

During the simulation, two different antenna designs were used to assess the imaging. A simple rectangular patch antenna is considered to serve as comparison to the final design. For distinguishing between healthy and pneumonia affected lungs, we used two $5.8 \mathrm{GHz}$ resonant frequency edgefed Vivaldi antenna based on [33]. The affordable and widely used FR4 material was used as a substrate in both designs.

For every imaging system we simulated the imaging system in CST using a Gaussian excitation signal and collected the Sparameters as a measure of the backscatter. In addition, the far field $3 \mathrm{D}$ plots and SAR values were studied to characterize the antenna and assess whether its radiation levels are within the official safety standards. Originally, we opted for a semirealistic shape for the human torso (elliptical cylinder in Fig. 9) with every layer having thickness values (in millimeter) as reported in Table II. Compared to the layers seen in Fig.7, minor changes were made to the model for sake of ease of modelling.

TABLE II. LENGH OF INDIVIDUAL LAYERS FOR SIMULATION OF ELLIPTICAL LUNG MODEL

\begin{tabular}{|c|c|c|c|c|c|c|c|c|c|}
\hline Skin & Fat & Muscle & Bone & Heart & Lung & Bone & Muscle & Fat & Skin \\
\hline 3 & 2 & 20 & 6 & 84 & 70 & 6 & 20 & 2 & 3 \\
\hline
\end{tabular}

Thickness values are given in millimeter. 


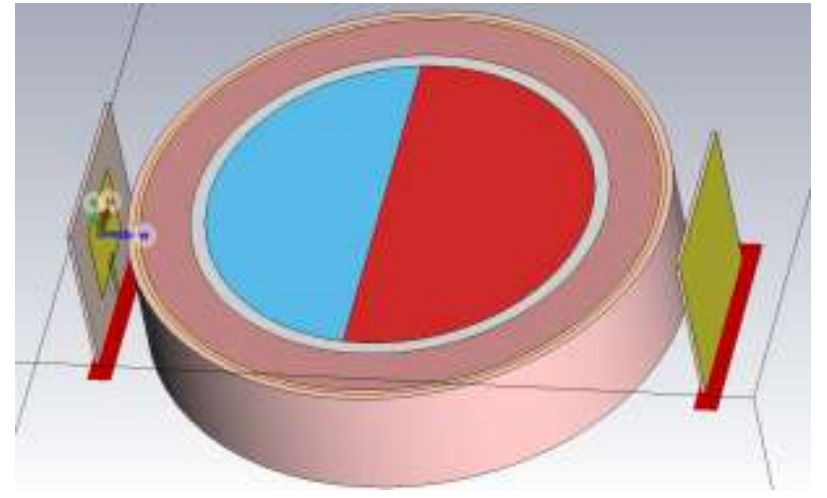

Fig. 9. Elliptical lung phantom model used with simple patch antenna.

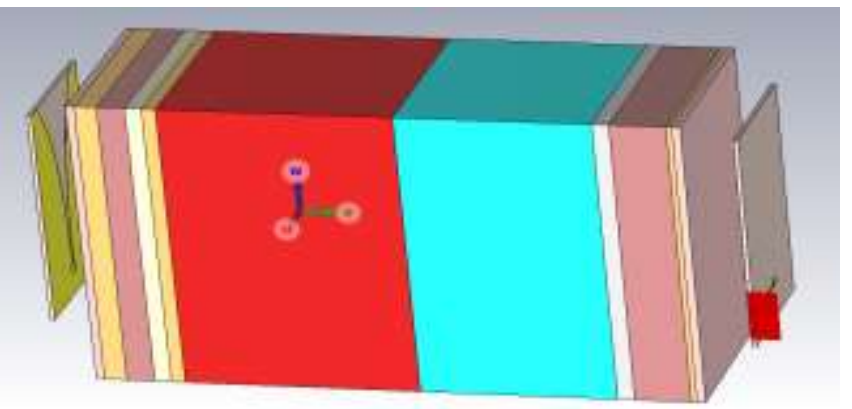

Fig. 10. Planar lung phantom model used with Vivaldi antenna based on [33].

However, the computation complexity for this model and the previous antenna design was beyond the capacity of the machine in use as it also had to calculate the large number of frequencies present in the antenna's frequency band and the corresponding dielectric constants for every human tissue layer. So the simpler patch antenna with a resonant frequency of $1.95 \mathrm{GHz}$ was used for this model. Its bandwidth is found to be $<200 \mathrm{MHz}$ so it does not qualify as ultrawide band.

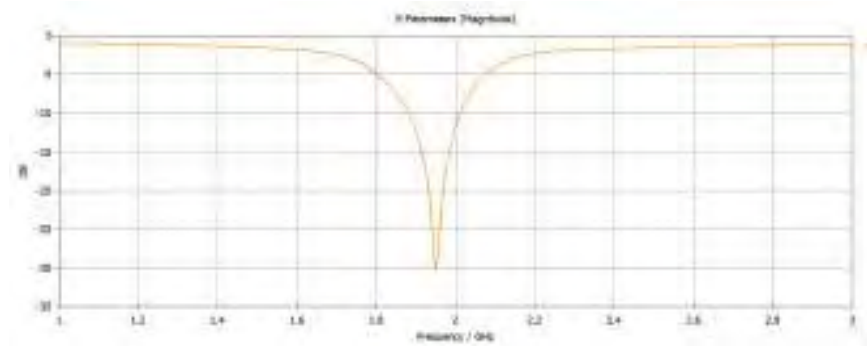

Fig. 11. Return loss of simple rectangular patch antenna.

TABLE III. Dimensions of Designed Antennas (IN Millimeter)

\begin{tabular}{|c|c|c|}
\hline \multicolumn{3}{|c|}{ Rectangular Patch } \\
\hline Patch Width & $\boldsymbol{W}$ & 36.27 \\
\hline Patch Length & $\boldsymbol{L}$ & 36.27 \\
\hline Ground plane width & $\boldsymbol{W g}$ & 72.54 \\
\hline Ground plane length & $\boldsymbol{L g}$ & 72.54 \\
\hline Feedline width & $\boldsymbol{W f}$ & 2.932 \\
\hline Feed inset & $\boldsymbol{F i}$ & 4.8 \\
\hline Substrate height & $\boldsymbol{h}$ & 1.6 \\
\hline Conductor height & $\boldsymbol{h t}$ & 0.035 \\
\hline
\end{tabular}

\begin{tabular}{|c|c|c|}
\hline \multicolumn{3}{|c|}{ Edge-fed Vivaldi } \\
\hline Slot line width & $\boldsymbol{W s}$ & 0.986 \\
\hline Throat width & $\boldsymbol{W m t}$ & 3.037 \\
\hline Coupler Width & $\boldsymbol{W m c}$ & 0.7623 \\
\hline Substrate height & $\boldsymbol{T}$ & 1.5748 \\
\hline Distance form cavity to coupler center & $\boldsymbol{S m c}$ & 0.3812 \\
\hline Strip line stub radius & $\boldsymbol{R} \boldsymbol{~}$ & 3.793 \\
\hline Conductor height & $\boldsymbol{M T}$ & 0.035 \\
\hline Width of strip line & $\boldsymbol{L s}$ & 0.762 \\
\hline Throat length & $\boldsymbol{L m t}$ & 10.32 \\
\hline Coupler Length & $\boldsymbol{L m c}$ & 4.913 \\
\hline Flare length & $\boldsymbol{L f}$ & 76 \\
\hline Taper width & $\boldsymbol{F t}$ & 40 \\
\hline Diameter of slot line cavity & $\boldsymbol{D c}$ & 4.913 \\
\hline
\end{tabular}

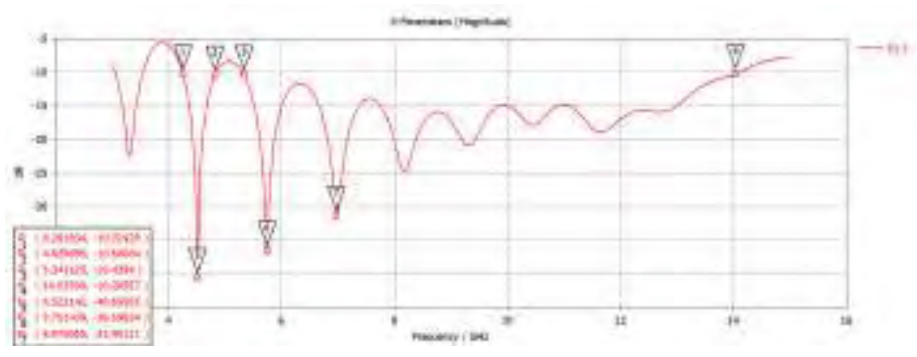

Fig. 12. Return loss of vivaldi antenna.

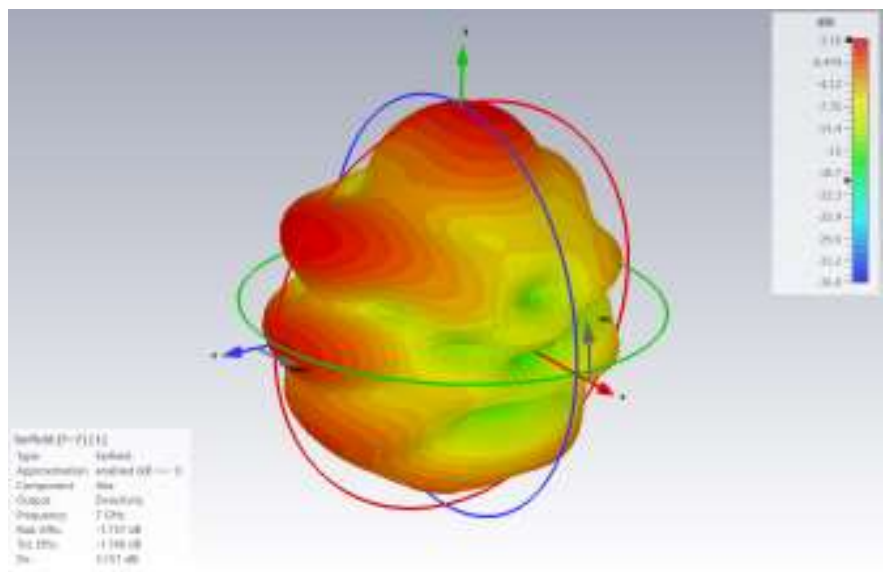

Fig. 13. Gain of vivaldi antenna at $7 \mathrm{GHz}$.

As shown in Fig. 10, the Vivaldi antennas were used with the simpler planar model, one of each antenna placed in front of the skin layer. The design parameters for the antennas are given in Table III. Fig. 12, 13 and 14 demonstrate the performance of Vivaldi antenna which is shown to be operating as an UWB antenna with resonant frequencies 4.5 $\mathrm{HHz}, 5.75 \mathrm{GHz}$ and $6.97 \approx 7 \mathrm{GHz}$. The maximum Specific absorption rate (SAR) of $0.971201 \mathrm{~W} / \mathrm{kg}$ is also well below the IEC 62209-1 limit of $2 \mathrm{~W} / \mathrm{kg}$ averaged over the $10 \mathrm{~g}$ of tissue, as demonstrated in Fig. 14. 


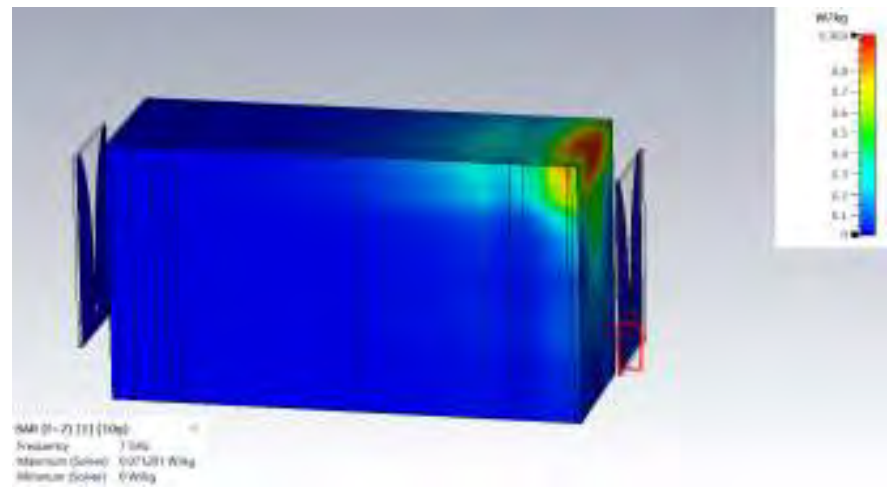

Fig. 14. SAR of vivaldi antenna at $7 \mathrm{GHz}$.

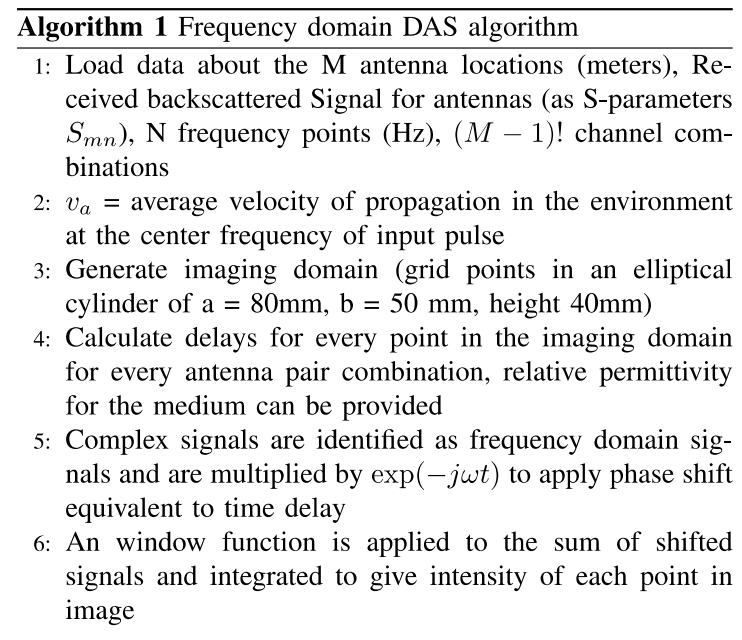

7: For each of the $\mathrm{M}$ antennas:

8: for $i=1$ to $M$ do

$S_{m, a v}(f)=\frac{1}{N} \sum S_{m n}(f) \quad \triangleright$ average S-parameter value over frequency

9: end for

$$
S_{m}(f)=S_{m n}(f)-S_{m, a v}(f) \quad \triangleright \text { noise removal }
$$

10: For each $(\mathrm{x}, \mathrm{y})$ point in $\mathrm{XY}$ imaging domain grid:

11: for $j=0$ to $X-1$ do

12: for $k=0$ to $Y-1$ do

$$
t_{m}(x, y)=\frac{2 d_{x, y}}{v_{a}}
$$

$\triangleright$ delay needed

for signal to travel from transmitter of mth antenna to the $(\mathrm{x}, \mathrm{y})$ point and back to the receiver of mth antenna

\section{3: $\quad$ end for}

14: end for

15: For each of the $M$ antennas:

16: for $i=1$ to $M$ do

17: $\quad$ Calculate $S_{m n}(f, x, y)=S_{m}(f) * \exp \left(-j \omega t_{m}(x, y)\right)$ $\triangleright$ phase shifting

18: end for

19: for $x=0$ to $X-1$ do

20: for $y=0$ to $Y-1$ do

$$
\begin{array}{lr}
S(f, x, y)=\frac{1}{M} \sum S_{m n}(f, x, y) & \\
Z(f, x, y)=W(f) * S(f, x, y) \quad \triangleright \text { Windowing } \\
I(x, y)=\int Z(f, x, y)^{2} d f \quad \triangleright \text { Calculate pixel }
\end{array}
$$

Fig. 15. Delay-and-Sum Algorithm implementation in MERIT.

The images were reconstructed by the DAS, CDAS and DMAS algorithms with the help of the open-source Microwave Radar-based Imaging Toolbox (MERIT) [34].

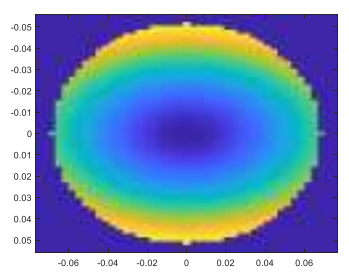

(a)

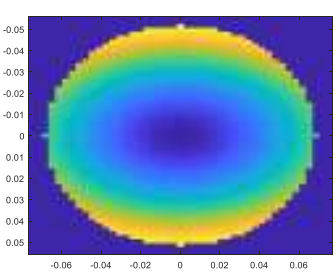

(b)

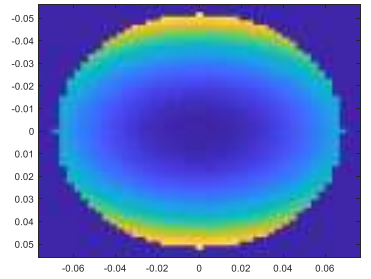

(c)

Fig. 16. Images found using rectangular antenna for DAS (a), CDAS (b) and DMAS (c)

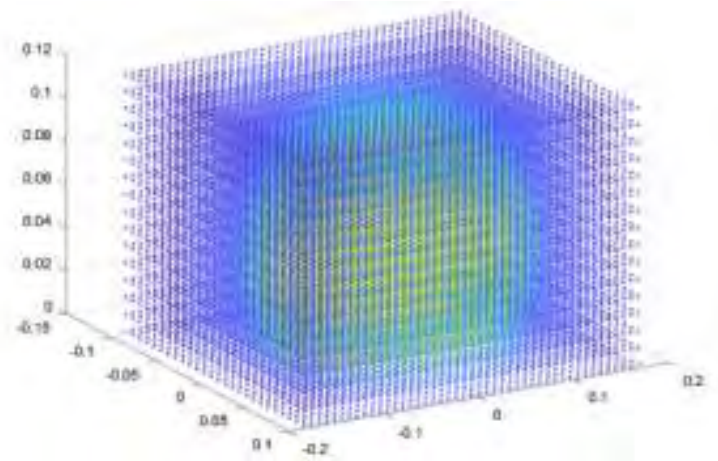

Fig. 17. Intensity values found using Vivaldi antenna as 3D scatter plot.

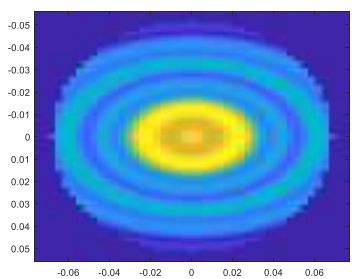

(a)

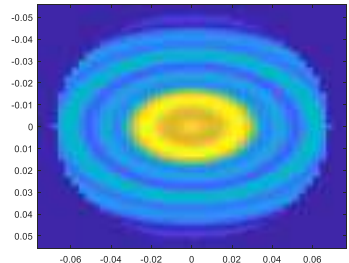

(c)

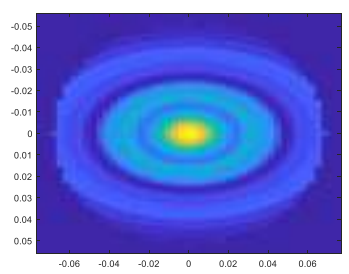

(e)

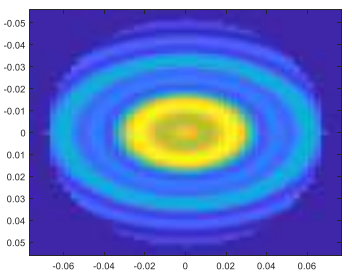

(b)

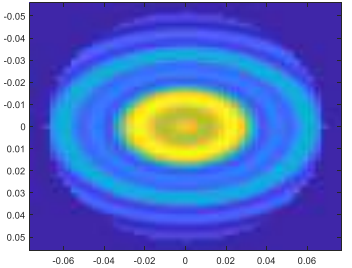

(d)

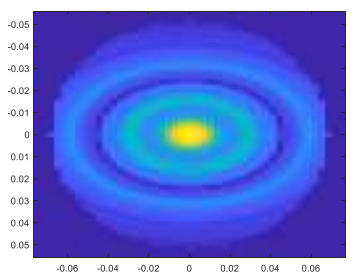

(f)
Fig. 18. Images found using Vivaldi antenna for DAS (a,b), CDAS (c,d) and DMAS (e,f) for normal lung (left) and lung with 50\% fluid (right). 
The S-parameter values were exported from MATLAB as ASCII text files. Then the information about antenna locations, the frequencies used for simulation and the complex values of the S-parameters were fed to MERIT using MATLAB. The intensity of the points of the different layers (regardless of whether the planar or the elliptical model was used) are mapped into an imaging domain, and a 2D cross section across xy plane taken for observation. For a healthy lung, the images in Fig. 17 and Fig. 18 (left column) are obtained. A number of rings are found in all pictures that correspond to the different tissue layer intersections. The brighter the image pixels in a ring, the more the contrast between the dielectric properties of the two layers surrounding it. The images generated with patch antenna have bright colors near the edges which imply a large amount of signal was backscattered from the skin layer, and the distinction between different layers is blurred. The rings are more evident in the images (Fig 18 (b), (d), and (f)) generated using the Vivaldi antenna due to its large bandwidth and resultant higher resolution. The images in Fig. 18 (right column) showing lung with higher fluid content have some visual differences compared to the ones for healthy lung. The amount of contrast is not very obvious visually so the immse() function in MATLAB has been used to calculate the mean-squared error (MSE) between the pixel data of healthy and unhealthy lung images. The mean-squared error is found to be 352.8194 , 193.0381 and 212.8439 respectively for DAS, CDAS and DMAS. While we have used frequency dependent body tissue layers in the model, we have not yet found any elegant way to extract that information for all frequencies and integrate the values into the delay calculation process between imaging point and antenna, which may be the reason for the low contrast.

\section{CONCLUSION}

The designed Vivaldi antenna has many attractive features like compact size, large bandwidth, and high directivity, but shows a low radiative efficiency which should be improved in future works. in the practical implementation of this work, we will aim to surround the torso with a wearable system comprising an antenna array rather than just two antennas. With a more powerful machine and access to laboratory equipment, more realistic and practical modelling of antennas would be possible. Another concern is the degree of difference found between healthy and pneumonia affected lung. So far we have used already existing popular algorithms, but the final goal of our work is to build a system using a novel algorithm that will accurately detect even the smallest change in the dielectric property of lung tissues. The literature review in Section II draws attention to the high detection accuracies reported by recent machine learning algorithms, but the limitations inherent to those methods imply that machine learning methods alone cannot provide a quick response to the rapidly growing need for prompt COVID-19 diagnosis. If microwave imaging methods, that are less expensive and more portable by nature, become widespread and integrated into medical imaging, then it possible that UWB imaging can work together with machine learning to provide quick radiological scans with little to no health risk.

\section{REFERENCES}

[1] L. Fan et al., "Progress and prospect on imaging diagnosis of COVID19," Chin. J. Acad. Radiol., vol. 3, no. 1, pp. 4-13, Mar. 2020, doi: 10.1007/s42058-020-00031-5.

[2] O. of the Commissioner, "Coronavirus Disease 2019 Testing Basics," FDA, Apr. 2021, Accessed: Jun. 13, 2021. [Online]. Available: https://www.fda.gov/consumers/consumer-updates/coronavirus-disease2019-testing-basics

[3] J. Vrieze, "Forget throat swabs: Dutch company claims its breathalyzer can help sniff out COVID-19," Science, May 2021, doi: 10.1126/science.abj5103.

[4] D. M. Ruszkiewicz et al., "Diagnosis of COVID-19 by analysis of breath with gas chromatography-ion mobility spectrometry - a feasibility study," EClinicalMedicine, vol. 29, Dec. 2020, doi: 10.1016/j.eclinm.2020.100609.

[5] W. Shao and T. McCollough, "Advances in Microwave Near-Field Imaging: Prototypes, Systems, and Applications," IEEE Microw. Mag., vol. 21, no. 5, pp. 94-119, May 2020, doi: 10.1109/MMM.2020.2971375.

[6] M. H. Fazalul Rahiman, T. Tan Wan Kiat, and P. J. Soh, "Microwave Tomography Application and Approaches - A Review," J. Teknol., vol. 73, Mar. 2015, doi: 10.11113/jt.v73.4258.

[7] E. Pancera, T. Zwick, and W. Wiesbeck, "Ultra wideband radar imaging: An approach to monitor the water accumulation in the human body," in 2010 IEEE International Conference on Wireless Information Technology and Systems, Honolulu, HI, USA, Aug. 2010, pp. 1-4. doi: 10.1109/ICWITS.2010.5611899.

[8] S. Dogu, İ. Dilman, M. Çayören, and İ. Akduman, "Imaging of pulmonary edema with microwaves - Preliminary investigation," in 2017 10th International Conference on Electrical and Electronics Engineering (ELECO), Nov. 2017, pp. 1054-1057.

[9] S. Ahdi Rezaeieh, "Wideband Microwave Imaging Systems for the Diagnosis of Fluid Accumulation in the Human Torso," PhD Thesis, The University of Queensland, 2016. doi: 10.14264/uq1.2016.452.

[10] N. Alam, A. Ahmed, M. A. I. Oni, T. A. Meem, and Md. A. Rahman, "UWB Microwave Imaging for Non-Invasive Anomaly Detection in Human Lung and Possible Application in COVID-19 Diagnosis: A Review," in 2021 2nd International Conference on Robotics, Electrical and Signal Processing Techniques (ICREST), Jan. 2021, pp. 772-776. doi: 10.1109/ICREST51555.2021.9331250.

[11] T. R. Niloy and A. Rahman, "Diagnosis of COVID-19 Infected Lungs from Chest X-ray Images using Deep Learning Algorithms," p. 8, 2021.

[12] L. Li et al., "Artificial Intelligence Distinguishes COVID-19 from Community Acquired Pneumonia on Chest CT," Radiology, Mar. 2020, doi: 10.1148/radiol.2020200905.

[13] X. Xu et al., "Deep Learning System to Screen Coronavirus Disease 2019 Pneumonia," ArXiv200209334 Phys., Feb. 2020, Accessed: Oct. 30, 2020. [Online]. Available: http://arxiv.org/abs/2002.09334

[14] Y. Song et al., "Deep learning Enables Accurate Diagnosis of Novel Coronavirus (COVID-19) with CT images," medRxiv, p. 2020.02.23.20026930, Feb. 2020, doi: 10.1101/2020.02.23.20026930.

[15] A. M. Hasan, M. M. AL-Jawad, H. A. Jalab, H. Shaiba, R. W. Ibrahim, and A. R. AL-Shamasneh, "Classification of Covid-19 Coronavirus, Pneumonia and Healthy Lungs in CT Scans Using Q-Deformed Entropy and Deep Learning Features," Entropy, vol. 22, no. 5, Art. no. 5, May 2020, doi: $10.3390 / \mathrm{e} 22050517$.

[16] L. Wang, Z. Q. Lin, and A. Wong, "COVID-Net: a tailored deep convolutional neural network design for detection of COVID-19 cases from chest X-ray images," Sci. Rep., vol. 10, no. 1, Art. no. 1, Nov. 2020, doi: 10.1038/s41598-020-76550-z.

[17] M. Turkoglu, "COVIDetectioNet: COVID-19 diagnosis system based on $\mathrm{X}$-ray images using features selected from pre-learned deep features ensemble," Appl. Intell., Sep. 2020, doi: 10.1007/s10489-020-01888-w.

[18] J. Zhang et al., "Viral Pneumonia Screening on Chest X-ray Images Using Confidence-Aware Anomaly Detection," ArXiv200312338 Cs Eess, Sep. 2020, Accessed: Oct. 30, 2020. [Online]. Available: http://arxiv.org/abs/2003.12338

[19] J. Li, D. Zhang, Q. Liu, R. Bu, and Q. Wei, "COVID-GATNet: A Deep Learning Framework for Screening of COVID-19 from Chest X-Ray 
Images," in 2020 IEEE 6th International Conference on Computer and Communications (ICCC), Dec. 2020, pp. 1897-1902. doi: 10.1109/ICCC51575.2020.9345005.

[20] S. Tabik et al., "COVIDGR Dataset and COVID-SDNet Methodology for Predicting COVID-19 Based on Chest X-Ray Images," IEEE J. Biomed. Health Inform., vol. 24, no. 12, pp. 3595-3605, Dec. 2020, doi: 10.1109/JBHI.2020.3037127.

[21] S. Wang et al., "A deep learning algorithm using CT images to screen for Corona Virus Disease (COVID-19)," medRxiv, p. 2020.02.14.20023028, Apr. 2020, doi: 10.1101/2020.02.14.20023028.

[22] T. Tuncer, F. Ozyurt, S. Dogan, and A. Subasi, "A novel Covid-19 and pneumonia classification method based on F-transform," Chemom. Intell. Lab. Syst., vol. 210, p. 104256, Mar. 2021, doi: 10.1016/j.chemolab.2021.104256.

[23] "Doses in Our Daily Lives," NRC Web. https://www.nrc.gov/aboutnrc/radiation/around-us/doses-daily-lives.html (accessed Jun. 15, 2021).

[24] New York state department "Radiation and Health." https://www.health.ny.gov/publications/4402.pdf (accessed Jun. 15, 2021).

[25] R. Cicchetti, E. Miozzi, and O. Testa, "Wideband and UWB Antennas for Wireless Applications: A Comprehensive Review," Int. J. Antennas Propag., vol. 2017, p. e2390808, Feb. 2017, doi: $10.1155 / 2017 / 2390808$.

[26] S. A. Rezaeieh, A. Zamani, K. S. Bialkowski, A. Mahmoud, and A. M. Abbosh, "Feasibility of Using Wideband Microwave System for NonInvasive Detection and Monitoring of Pulmonary Oedema," Sci. Rep., vol. 5, Sep. 2015, doi: 10.1038/srep14047.

[27] F. Wang, T. Arslan, and G. Wang, "Breast cancer detection with microwave imaging system using wearable conformal antenna arrays," in 2017 IEEE International Conference on Imaging Systems and Techniques (IST), Oct. 2017, pp. 1-6. doi: 10.1109/IST.2017.8261547.

[28] M. M. Abdelhamid and A. M. Allam, "Detection of lung cancer using ultra wide band antenna," in 2016 Loughborough Antennas Propagation Conference (LAPC), Nov. 2016, pp. 1-5. doi: 10.1109/LAPC.2016.7807452.

[29] M. T. Islam, M. Z. Mahmud, M. T. Islam, S. Kibria, and M. Samsuzzaman, "A Low Cost and Portable Microwave Imaging System for Breast Tumor Detection Using UWB Directional Antenna array," Sci. Rep., vol. 9, no. 1, Art. no. 1, Oct. 2019, doi: 10.1038/s41598-01951620-z.

[30] M. Cavagnaro, E. Pittella, and S. Pisa, "UWB pulse propagation into human tissues," Phys. Med. Biol., vol. 58, no. 24, pp. 8689-8707, Dec. 2013, doi: 10.1088/0031-9155/58/24/8689.

[31] T. Yamashiro, M. Ando, Y. Okazaki, and S. Sasaguri, "Dielectric behavior of pulmonary edema induced in the rat lung," Respir. Physiol. Neurobiol., vol. 145, no. 1, pp. 91-100, Jan. 2005, doi: 10.1016/j.resp.2004.08.008.

[32] P. Nopp, E. Rapp, H. Pfutzner, H. Nakesch, and C. Rusham, "Dielectric properties of lung tissue as a function of air content," Phys. Med. Biol., vol. 38, no. 6, pp. 699-716, Jun. 1993, doi: 10.1088/0031$9155 / 38 / 6 / 005$

[33] "EM Simulation Models," Z. PENG, Dec. 03, 2020. https://zpeng.me/index.php/em-simulation-models/ (accessed Jun. 16, 2021).

[34] D. O'Loughlin et al., "Open-source Software for Microwave Radarbased Image Reconstruction," in 12th European Conference on Antennas and Propagation (EuCAP 2018), London, UK, 2018, p. 408 (4 pp.)-408 (4 pp.). doi: 10.1049/cp.2018.0767.

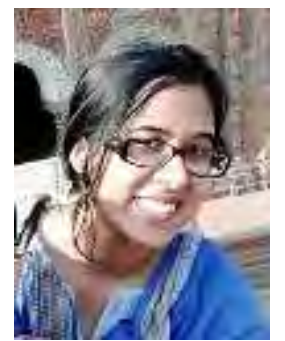

Nowshin Alam is an Assistant Professor of the department of Electrical and Electronic Engineering, American International University-Bangladesh since 2018. She received her Bachelor of Science degree in Electrical and Electronic Engineering from Bangladesh University of Engineering and
Technology (BUET) and Master of Science degree in Electrical Engineering from University of California, Riverside (UCR), in year 2014 and 2016 respectively. She started her career as a System Engineer at Huawei Technologies Ltd., Bangladesh since September 2014 and served there till April 2016. She joined academia after getting her MSc degree and briefly worked at Green University, Dhaka as a lecturer in Electrical Engineering from May 2018 to August 2018.

Her research interest includes Wireless Communications, Signal Processing, Solar Cell Design, Machine Learning and Antenna Propagation. In her free time, she likes to write articles and create youtube videos on efficient online teaching, increasing productivity through automation and programming, and applying technological approaches to education.

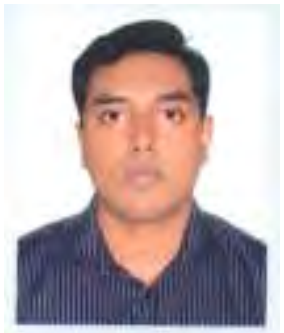

Md. Abdur Rahman is faculty member of the faculty of engineering since 2002 at American International UniversityBangladesh (AIUB). Currently he is employed as Professor and Associate Dean of the Faculty of Engineering in the same university. He received $\mathrm{PhD}$ in wireless communications from Tokyo Institute of Technology, Japan in 2013.

$\mathrm{He}$ received BSc Engg. and M.E. degrees from American International University Bangladesh (AIUB) and Asian Institute of Technology (AIT), Thailand, respectively in 2002 and 2006. His recent research interests include cognitive radio, bioinformatics, wireless systems, ICT etc. He conducted postdoctoral research in the School of Engg. and IT at Federation University Australia on Bio-informatics. He is a Life member of IEB and senior member of IEEE. During his doctoral studies he lead a team of students formed by both undergraduate and postgraduate students for designing an emergency wireless network. The team took part in four international research competitions and won four prizes including Wireless Innovation Smart Radio Challenge, IEEE president's change the world outstanding humanitarian award, IEEE DSPS best student presentation and IEEE Tokyotech Best Paper award. Currently he is residing in Dhaka, Bangladesh and working on the improvement of teaching learning methods along with innovative engineering projects. 